\title{
Spontaneous subconjunctival and retinal hemorrhage in acute on chronic liver failure: A case report and brief review of the literature
}

\author{
Himanshu Gupta ${ }^{1}$; Prasanta Debnath ${ }^{2}$; Akash Roy ${ }^{*}$ \\ ${ }^{1}$ Department of Hepatology, Sanjay Gandhi Post Graduate Institute of Medical Sciences (SGPGIMS), Lucknow, India. \\ ${ }^{2}$ Department of Gastroenterology, TNMC \& BYL Nair Charitable Hospital, Mumbai.
}

\section{*Corresponding Author: Akash Roy}

Assistant Professor, Department of Hepatology,

SGPGIMS, Lucknow, India.

Email: royakash12@gmail.com

Received: Jun 28, 2021

Accepted: Aug 13, 2021

Published: Aug 17, 2021

Archived: www.jjgastro.com

Copyright: (C) Roy A (2021).

\section{Clinical case}

A 48-year-old male with a history of significant alcohol consumption (120 gm per day for six years) and a recent binge presented with jaundice for one month followed by abdominal distention for ten days. At presentation, he was normotensive (BP 110/70 mmHg), had tachycardia (HR 122/min), and maintained saturation at room air $\left(\mathrm{SpO}_{2} 98 \%\right)$. Physical examination was remarkable for scleral icterus, palmar erythema, enlarged liver (span $14 \mathrm{~cm}$ ), and free fluid in the abdomen. Laboratory investigations revealed anemia (Hemoglobin $10.1 \mathrm{gm} \%$ ), leucocytosis (White blood cell count $17000 / \mathrm{mm}^{3}$ ), thrombocytopenia (Platelet count $90 \times 103) / \mathrm{mm}^{3}$ ), deranged liver function tests (Total bilirubin $9.5 \mathrm{gm} / \mathrm{dl}$, Alanine Transaminase $52 \mathrm{U} / \mathrm{L}$, Aspartate Transaminase (112 U/L), and coagulopathy (International normalized ratio 2.2, Prothrombin time $20 \mathrm{sec}$ ). A provisional diagnosis of Acute on Chronic Live Failure (ACLF) with severe alcoholic hepatitis as the precipitating event was made. The patient was started on standard therapy with nutritional supplementation, diuretics, laxatives. On day 4 of admission, the patient developed bilateral red-eye. There was no history of any diminution of vision, double diplopia, ocular discharge, orbital pain, antecedent trauma, cough, or any interventional procedure. Vision, pupillary reflexes, intraocular pressure as well as range of extraocular movements were normal.

Opthalmology opinion was consistent with bilateral subconjunctival hemorrhage with associated retinal hemorrhages (Figure $1 a, b)$. The patient was managed conservatively with blood product substitution for correction of coagulopathy. Resolution and disappearance of hemorrhagic changes were noted on follow-up after 28 days. 
Citation: Gupta H, Debnath P, Roy A. Spontaneous subconjunctival and retinal hemorrhage in acute on chronic liver failure: A case report and brief review of the literature. Japanese J Gastroenterol Res. 2021; 1(3): 1012.
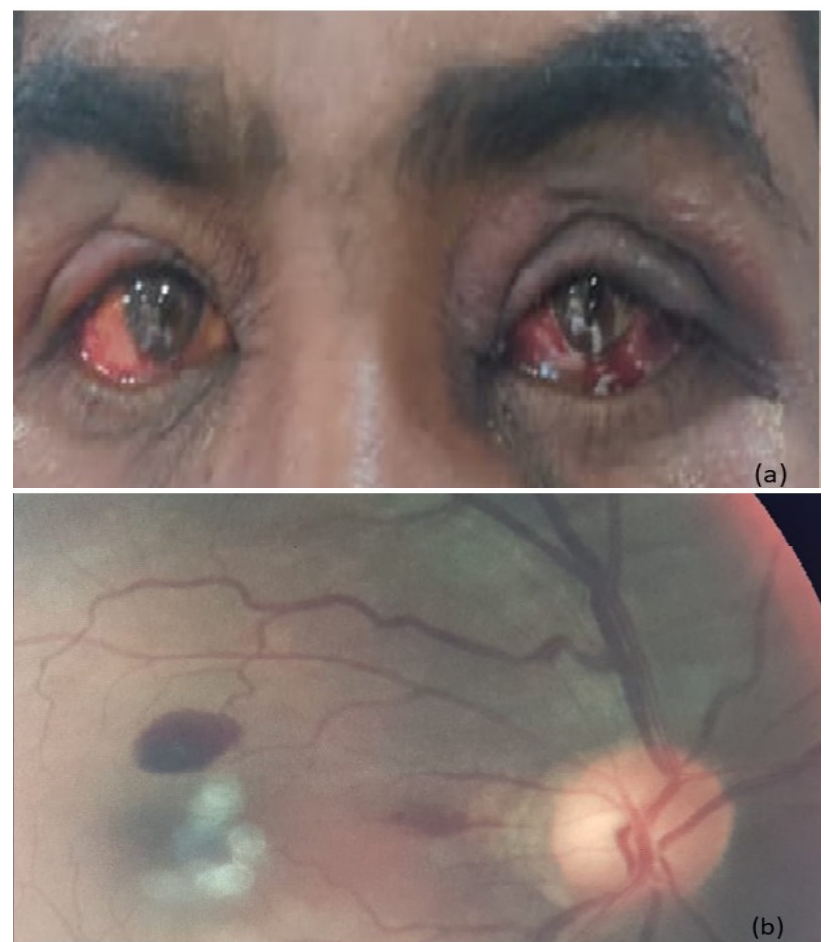

Figure 1: (a) Showing bilateral subconjunctival hemorrhage (b) Fundoscopy showing retinal hemorrhage

\section{Discussion}

Coagulopathy and hematological dyscrasias are potential risk factors for subconjunctival and retinal hemorrhages [1]. Patients with acute on chronic liver failure are frequently coagulopathic and are thus theoretically prone to such hemorrhagic changes. We reviewed the literature on subconjunctival and retinal hemorrhages in cirrhosis and found that though intuitively expected, such changes have not been commonly reported. Elaraoud et al. and Ness et al. have reported two individual cases of multilayered retinal hemorrhage and spontaneous orbital hemorrhage, respectively, in patients with alcoholic cirrhosis $[2,3]$. In both cases, the presumed aggravating factor was cough which could have lead to sudden venous congestion and precipitation of hemorrhage in a setting of coagulopathy.
The other setting which has been recently described is the development of subconjunctival hemorrhage after endoscopy in a patient with portal hypertension, which was attributed to periprocedural retching and transient increase in intrathoracic pressure [4]. In all the described cases, there was a spontaneous resolution of the hemorrhage with only the requirement of coagulopathy correction. To the best of our knowledge, this represents a unique case in which there was a spontaneous development of concomitant subconjunctival and retinal hemorrhage occurring without any precipitating factor. Such an observation draws home the point that patients with coagulopathic states like ACLF are susceptible to spontaneous ocular hemorrhages, which usually tend to subside with correction of coagulopathy.

\section{Declarations}

Ethics approval and consent to participate: Written informed consent obtained from the patient.

Consent for publication: Written informed consent obtained from the patient.

Availability of data and material: Yes.

Authors' contributions: HG: Data compilation; PD: critical revision; AR Writing and Critical Revision.

\section{References}

1. Tarlan B, Kiratli H. Subconjunctival hemorrhage: risk factors and potential indicators. Clinical ophthalmology (Auckland, NZ). 2013; 7: 1163.

2. Elaraoud I, Damato E. Bilateral extensive multiple multi-layered retinal haemorrhage in a patient with alcoholic liver disease. The Pan African Medical Journal. 2018; 29.

3. Ness C, Sakpal SV. Cirrhosis induced spontaneous orbital hemorrhage. Digestive and Liver Disease. 2020; 52: 922-923.

4. Rajvanshi P, McDonald GB. Subconjunctival hemorrhage as a complication of endoscopy. Gastrointestinal endoscopy. 2001; 53: 251-253. 